\section{TUBERCULOUS MENINGITIS: A RARE AND SEVERE FORM OF AN EMERGING DISEASE?}

M. Cabral, L. Varandas, C. Gouveia, M. Conde, M.J. Brito

Pediatric Infectious Diseases Unit, Pediatric Department, Hospital Dona Estefânia; Centro Hospitalar Lisboa Central, Lisbon, Portugal

Background and aims: Tuberculous meningitis (TBM) accounts for $0.7 \%$ of all cases of tuberculosis (TB). The incidence is related to the prevalence of TB in the community. In Portugal BCG vaccine is routinely administered and has been proven to reduce the incidence of severe disseminated disease in children.

Methods: Retrospective study, between January 2000 and December 2010, of children with TBM. Diagnosis confirmed by culture and/or polymerase chain reaction of the CSF or cerebral tissue.

Results: 5 patients, mean 4.2 years old ( 9 months -10 years); one of african origin. All children were immunized against Mycobacterium tuberculosis. Fever was the first clinical manifestation in four children, accompanied by vomiting (5), malaise (3), lethargy (5), headache (2), cough (2), with more than two weeks in duration in three. One had concomitant miliary disease. Tuberculin skin test was positive only in one and all had contact with patients with TB. There was one multidrug-resistant TBM. First line agents were replaced in two patients because of oral intolerance and antimicrobial resistance. 4/5 required intensive care. Complications occurred in all cases: seizures (3), coma (3), cranial nerve paralysis (2), cerebral stroke (1), motor disabilities (3), hydrocephalus (5) and venous thrombosis (1). One child died, whose diagnosis was made postmortem.

Conclusion: TB remains among the great public health challenges. It should be considered when diagnosing children with nonpecific/subacute symptoms of neurologic infection, even in absence of respiratory symptoms or an epidemiologic context. Death may occur as a result of missed diagnosis and delayed treatment.
886

\section{ROUTINE MALE CIRCUMCISION IN THE YOUNG: IS IT TIME TO RECONSIDER THE BENEFITS?}

\author{
D. Liư ${ }^{1}$, A. Patil ${ }^{2}$, T. Ariyanayagam³,
} M. Mulchandani ${ }^{4}$

${ }^{1}$ Imperial College School of Medicine, ${ }^{2}$ Barts \& The London School of Medicine and Dentistry, ${ }^{3} \mathrm{UCL}$ Medical School, London, ${ }^{4}$ Royal United Hospital, London, UK

Background: Male Circumcision (MC) has been a surgical intervention for thousands of years but only recently have randomised controlled trials (RCTs) been available to demonstrate a protective effect from sexually transmitted infections (STIs) most notably including HIV.

Aims: To analyse current available literature on neonatal $\mathrm{MC}$ and evaluate its routine implementation in infants and neonates.

Methods: Publications dealing with routine male circumcision using PubMed were reviewed. MeSH terms "male circumcision" were searched with additional terms "neonate", "infant", "newborn" and "routine".

Results: Recent evidence clarifies that $M C$ is completely protective against specific prepucial disease including phimoses and genital dermatoses. There is also strong RCT-based literature that MC is efficacious in the reduction of risk associated with female-to-male/insertive STIs. The protection may extend to female partners of circumcised men but this is observational and not unequivocal. Evidence for $\mathrm{MC}$ relating to genital malignancy in both men and women is also of limited power but appears to favour circumcision as protective. Studies demonstrate that $\mathrm{MC}$ is not however without a small risk of minor complications ( $0.2 \%)$ and there remains questions over the mechanism of protection as well as longterm bio-psycho-social sequelae.

Conclusions: The advantages for routine $\mathrm{MC}$ in the young include easily treatable diseases in the developed world. Penile cancer and HIV are some of the more serious illnesses associated but these are modifiable by genital hygiene and sexual behaviour. Unless there is a definitive reason for MC there is no case for a change in conservative practice as a result of recent literature. 